\title{
(1) Celiac Crisis in a Young Woman: Raising Awareness of a Life-Threatening Condition
}

\author{
Maryam Jameshorani ${ }^{1}$, Akram Pourshams $^{2}$, Anahita Sadeghi ${ }^{1{ }^{*},}$, Hiva Saffar ${ }^{3}$, Reza Malekzadeh ${ }^{2}$
}

1. Zanjan University of Medical Sciences. Tehran, Iran

2. Disease Research Center, Digestive Disease Research Institute, Tehran University of Medical Sciences, Shariati Hospital, Tehran, Iran

3. Department of Pathology, Shariati Hospital, Tehran University of Medical Sciences, Tehran, Iran

\section{* Corresponding Author: \\ Anahita Sadeghi, MD \\ Assistant Professor of Internal Medicine, Digestive Disease Research Center, \\ Digestive Disease Research Institute, Tehran University of Medical Sciences, Shariati Hospital, North Kargar Avenue, Tehran, Iran \\ Tel: + 982182415104 \\ Fax: + 982182415400 \\ Email: anahita825@gmail.com}

Received: 28 Apr. 2019

Accepted: 26 Aug. 2019

\begin{abstract}
Celiac crisis is a rare, acute, and life-threatening presentation of celiac disease. Its clinical presentations consist of severe watery non-bloody diarrhea, electrolyte disturbances (i.e. hypokalemia, hyponatremia, hypomagnesemia, hypocalcemia, and metabolic acidosis), hypoproteinemia, and dehydration. Here we present a 33-year-old woman who referred with profuse diarrhea, weight loss, hemodynamic instability, hypokalemia, hypoproteinemia, ascites, pancytopenia, and iron deficiency anemia. She used herbal medicines for constipation and had severe weakness after her childbirth. The patient was diagnosed as having celiac disease through pathological and serological evaluations 10 months earlier.

Diagnosis of celiac crisis after ruling out the other causes of resistant celiac was made and she was treated with steroids.
\end{abstract}

KEYWORDS:

Celiac crisis, Celiac disease, Diarrhea

Please cite this paper as:

Jameshorani M, Pourshams A, Sadeghi A, Saffar H, Malekzadeh R. A Celiac Crisis in a Young Woman: Raising Awareness of a Life-Threatening Condition. Middle East J Dig Dis 2019;11:230-233. doi:10.15171/mejdd.2019.154.

\section{INTRODUCTION}

Celiac disease (CD) is an immune-mediated enteropathy triggered by an inappropriate response to dietary gluten and characterized by malabsorption and villous atrophy. ${ }^{1}$ Celiac crisis has been used to describe the acute and fulminant form of $\mathrm{CD}$ associated with hypoproteinemia and edema. ${ }^{2}$ Other clinical presentations consist of severe diarrhea, electrolytes disturbances (hypokalemia, hyponatremia, hypomagnesemia, hypocalcemia, and metabolic acidosis), hypoproteinemia, and dehydration. ${ }^{3}$ Celiac crisis is a rare condition that has nearly disappeared from our experience. ${ }^{4}$ Although most of the cases of celiac crisis are presented in childhood, it should be considered in adults presenting with acute severe diarrheal illness, electrolyte disturbances, and malabsorption. ${ }^{5} \mathrm{CD}$ rarely presents with life-threatening complications, but patients who are diagnosed with the celiac crisis are associated with high morbidity. ${ }^{6}$ In almost all cases hospital admission and correction of metabolic and electrolytes imbalances with intravenous fluids are required, and in some cases corticotherapy is 
necessary. ${ }^{7}$ The mainstays of treatment of celiac crisis are the initiation of a gluten-free diet, parenteral fluid replacement, and nutritional support, and in most cases corticosteroids. ${ }^{8}$ In order to improve the awareness of clinicians about the celiac crisis and to facilitate its diagnosis, we reviewed a case of celiac crisis in a university-affiliated hospital.

\section{CASE REPORT}

A 33-year-old woman with a 10-month history of $C D$ was admitted to the hospital because of severe watery non-bloody diarrhea and weakness. Her symptoms began after second childbirth about 11 months earlier demonstrating with weakness and constipation. Then, according to her family's recommendation, she was treated with herbal remedies (mixture herbal products) and gradually suffered from weight loss, watery diarrhea, and generalized edema. She was admitted to a local hospital and underwent diagnostic and therapeutic approaches. According to the high titers of IgA anti-tissue transglutaminase antibodies $(>200 \mathrm{U} / \mathrm{mL}$, normal: < $10 \mathrm{U} / \mathrm{mL}$ ) and duodenal biopsies compatible with moderate to severe malabsorption and positive HLA-DQ2, and DQ8, she was diagnosed as having $\mathrm{CD}$ and treated with gluten-free diet for two months before admission. Her general condition improved for a short time, but diarrhea, weakness, $10 \mathrm{~kg}$ weight loss, and edema of the organs came back again after 2 months and she was admitted to our hospital.

The physical examination revealed hypotension, mild confusion, fever, minimal pain in the palpation of the lower abdominal quadrants, moderate ascites, and pitting edema in her legs.

Laboratory values showed pancytopenia (WBC:1500 mm³, hemoglobin $(\mathrm{Hb}): 7 \mathrm{~g} / \mathrm{dL} \mathrm{MCV}$ $68 \mathrm{fL}$, and Platelet count:170000), hypokalemia (K: $2.5 \mathrm{mmol} / \mathrm{L}$ ), hypocalcemia (Ca: $6.5 \mathrm{ng} / \mathrm{mL}$ ), hypophosphatemia ( $p: 1 \mathrm{ng} / \mathrm{mL})$, hypoalbuminemia $(3.1 \mathrm{~g} / \mathrm{L})$, iron $29 \mu \mathrm{g} / \mathrm{dL}$, ferritin $8.6 \mathrm{ng} / \mathrm{mL}$, hepatic enzymes dysfunction (AST: $185 \mathrm{U} / \mathrm{L}$, ALT: 156 $\mathrm{U} / \mathrm{L}$, ALK $p$ : $1193 \mathrm{U} / \mathrm{L}$, total bilirubin: $1.8 \mathrm{mg} / \mathrm{dL}$, direct bilirubin: $1 \mathrm{mg} / \mathrm{dL}$, prothrombin time: 16.5 s), and CRP: $4.3 \mathrm{mg} / \mathrm{dL}$. Vitamin B12 and folate levels were mildly low. She had normal renal and thyroid function. Stool microscopy and culture on three stool specimens were normal. Bone marrow aspiration and biopsy were normal. Brain computed tomography (CT) showed mild hydrocephalus. CT enterography revealed a reduction of jejunum folding and jejunal wall thickness with increased mucosal enhancement, multiple mesenteric LAP ascites, and pleural effusion. IgA anti-tissue transglutaminase antibodies were high.

Laparoscopic biopsies of jejunum showed acute and chronic inflammation both PMN and lymphocyte in lamina propria layer), but there was no evidence of malignancy. Liver biopsy showed severe steatosis. The liver and small intestine biopsy samples were reviewed by two expert gastrointestinal pathologists (figures 1, 2). Sections from liver wedge biopsy revealed steatohepatitis (grade: 5 and stage: 1 based on Brunt and Tiniakos classification. Portal inflammation was a little higher than could be expected in usual steatohepatitis. It could be explained by using herbal medicine or taking parenteral nutrition. Also, findings from intestinal biopsy showed a malabsorptive pattern, which was consistent with flare up of disease.

On the admission, she was resuscitated with intravenous fluids, total parenteral nutrition, potassium chloride, albumin, calcium, and phosphate supplements.

She was diagnosed as having celiac crisis and went well on a gluten-free diet, intravenous corticosteroid, and broad-spectrum antibiotics. She had a gradual recovery, and diarrhea and edema decreased in the first weeks of treatment and electrolyte abnormalities and pancytopenia were corrected. Unfortunately, at the fourth week of treatment, she suffered from sudden onset fever, dyspnea, and productive cough. The symptoms progressed and she was intubated with subsequent mechanical ventilation and transferred to intensive care unit (ICU). Despite all medical treatment, she died on the third day of the ICU admission.

\section{DISCUSSION}

Celiac crisis is a life-threatening condition that presents with severe diarrhea, hypoproteinemia, metabolic/ 


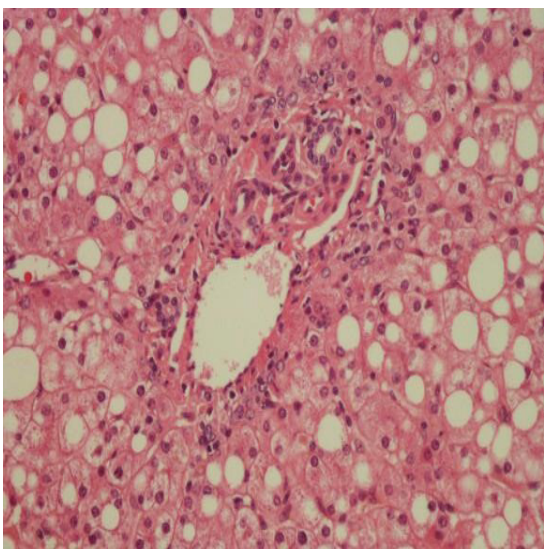

Fig.1: Liver biopsy showing pan lobular macrosteatosis associated with mild portal inflammation.
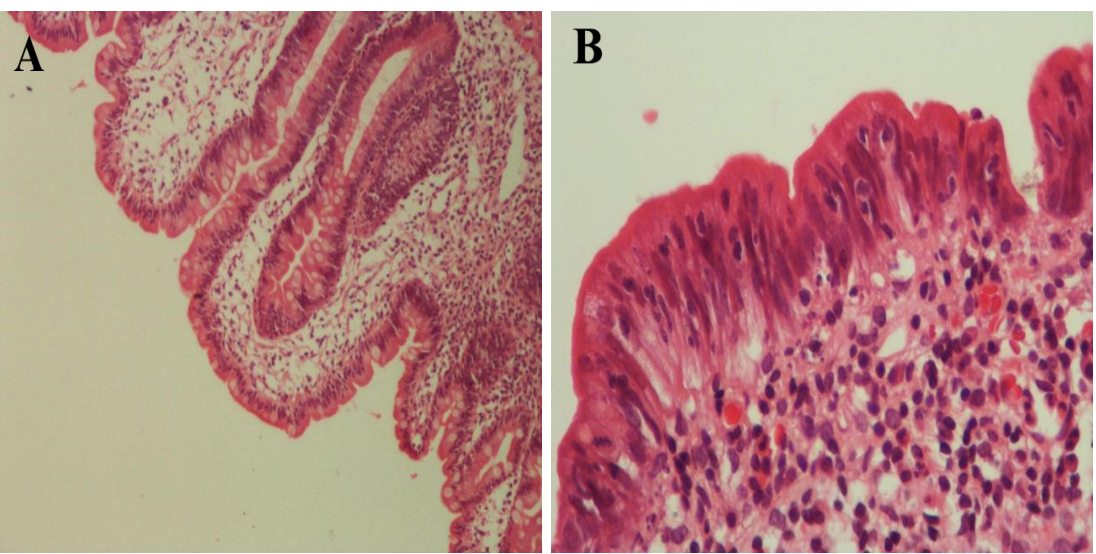

Fig.2: (A) Intestinal mucosa with villous blunting and (B) increased number of intraepithelial lymphocytes. electrolyte disturbances, and hypoproteinemia.9,10 Celiac crisis has a high morbidity, although it rarely occurs in adults and often has clear precipitating factors. ${ }^{11}$ The treatment of celiac crisis is gluten-free diet, but some patients require steroids. ${ }^{12}$ It is not clear why the celiac crisis has become a rare event during the past 50 years. It may be because of the changing nature of $\mathrm{CD}$ in general (ie, toward a more subtle and indolent disease). ${ }^{4}$

Proposed criteria for its definition require the acute onset or rapid progression of gastrointestinal symptoms (severe diarrhea, vomiting), attributable to the $\mathrm{CD}$ requiring hospital admission and/or nutrition with at least two of the following criteria: ${ }^{13}$

1. Signs of severe dehydration (hemodynamic instability and/or orthostatic changes);

2. Neurological dysfunction (peripheral neuropathy and tetany due to hypocalcemia);

3. Renal dysfunction (creatinine $>176.8 \mu \mathrm{mol} / \mathrm{L}$ or $2.0 \mathrm{~g} / \mathrm{dL}$ );

4. Metabolic acidosis $(\mathrm{pH}<7.35)$;

5. Albumin $<3.0 \mathrm{~g} / \mathrm{dL}$;

6. Electrolyte disturbances (hypokalemia, hyper/ hyponatremia, hypocalcemia, hypomagnesemia);

7. Weight loss $>10 \mathrm{lbs}(4.5 \mathrm{Kg})$

In this clinical case, the patient had five of these criteria. She was initially treated with gluten-free diet, and in the course of her disease, corticosteroid was added. It has been shown in several studies that some individuals develop celiac crisis, contrary to the most of adult patients who present a mild course of the disease. ${ }^{14}$ In most cases, an immune stimulus (infection, severe inflammation, surgery, pregnancy, or not following a gluten-free diet) happened prior to the crisis, which leads to the hypothesis that a combination of activation of the immune system, severe mucosal inflammation, and disruption of normal intestinal motility could be involved. ${ }^{12}$

Although the cause of our patient's condition is unclear, it may be due to herbal remedies and infections.

In summary, it is important to consider that $\mathrm{CD}$ may present with "crisis" in patients who present with the acute onset of severe diarrhea and hypoproteinemia with or even without a history of CD. Due to its high morbidity rate, prompt diagnosis and treatment is crucial. Intestinal biopsies in cases caused by infections or those secondary to protein allergy may show non-specific villous atrophy and crypt hyperplasia and may be indistinguishable from biopsies of patients with CD. Corticosteroids and appropriate antibiotic coverage should be considered in celiac crisis when a rapid response to a gluten-free diet does not occur. Nutritional support, fluid and electrolyte repletion is often required in the short term too.

\section{ETHICAL APPROVAL}

There is nothing to be declared.

\section{CONFLICT OF INTEREST}

The authors declare no conflict of interest related to this work.

\section{ACKNOWLEDGEMENT:}

I would like to thank Professor Masoud Sotoudeh, the clinical pathologist, for reviewing the liver and 
small intestine biopsies that greatly improved the diagnostic evaluation.

\section{REFERENCES}

1. Green PH, Cellier C. Celiac disease. $N$ Engl J Med 2007;357:1731-43. doi:10.1056/NEJMra071600.

2. Fasano A, Catassi C. Current approaches to diagnosis and treatment of celiac disease: an evolving spectrum. Gastroenterology 2001;120: 636-51. doi:10.1053/gast.2001.22123.

3. Ferreira R, Pina R, Cunha N, Carvalho A. A celiac crisis in an adult: raising awareness of a lifethreatening condition. Eur J Case Rep Intern Med. 2016;3:000384. doi: 10.12890/2016_000384.

4. Mones RL, Atienza KV, Youssef NN, Verga B, Mercer GO, Rosh JR. Celiac crisis in the modern era. J Pediatr Gastroenterol Nutr 2007;45:480-3. doi:10.1097/MPG.0b013e318032c8e7.

5. Mrad RA, Ghaddara HA, Green PH, El-Majzoub N, Barada KA. Celiac Crisis in a 64-Year-Old Woman: An Unusual Cause of Severe Diarrhea, Acidosis, and Malabsorption. ACG Case Rep J 2015;2:95-7. doi: 10.14309/crj.2015.16.

6. Reilly NR, Fasano A, Green PH. Presentation of celiac disease. Gastrointest Endosc Clin $N$ Am 2012;22:613-21. doi: 10.1016/j.giec.2012.07.008.

7. Bai JC, Fried M, Roberto G. Celiac disease-World Gastroenterology Organisation Global Guidelines . World Gastroenterol Organ 2012:1-5.

8. Gupta T, Mandot A, Desai D, Abraham P, Joshi A. Celiac crisis with hypokalemic paralysis in a young lady. J Gastroenterol 25:259-60.

9. Fasano A, Catassi C. Current approaches to diagnosis and treatment of celiac disease: an evolving spectrum. Gastroenterology 2001;120:636-51. doi: 10.1053/ gast.2001.22123.

10. Rubio-Tapia A, Kyle RA, Kaplan EL, Johnson DR, Page W, Erdtmann F, et al. Increased Prevalence and Mortality in Undiagnosed Celiac Disease. Gastroenterology 2009;137:88-93. doi: 10.1053/j. gastro.2009.03.059.

11. Atikou A, Rabhi M, Hidani H, El Alaoui Faris M, Toloune F. Coeliac crisis with quadriplegia due to potassium depletion as presenting feature of coeliac disease. Rev Med Interne 2009;30:516-8. doi: 10.1016/j.revmed.2008.11.012.

12. Jamma S, Rubio-Tapia A, Kelly CP, Murray J, Najarian $\mathrm{R}$, Sheth $\mathrm{S}$, et al. Celiac crisis is a rare but serious complication of celiac disease in adults. Clin Gastroenterol Hepatol 2010;8:587-90. doi: 10.1016/j. cgh.2010.04.009.

13. Magro R, Pullicino E. Coeliac crisis with severe hypokalemia in an adult. Malta Med J 2012;24:36-8.
14. Bürk K, Farecki ML, Lamprecht G, Roth G, Decker $\mathrm{P}$, Weller $\mathrm{M}$, et al. Neurological symptoms in patients with biopsy-proven celiac disease. Mov Disord 2009; 24:2358-62. doi:10.1002/mds.22821. 